\title{
SYMPLECTIC GEOMETRY
}

\author{
BY ALAN WEINSTEIN
}

0. Introduction. Classical mechanics in the time of Huygens (1629-1695) and Newton (1642-1727) was very geometrical. Although Newton invented the calculus in order to formulate and solve physical problems, many of his arguments made heavy use of euclidean geometry. After Newton, there came a period of "mécanique analytique," during which Lagrange (1736-1813) could boast that his treatise on mechanics contained no pictures. ${ }^{1}$ Following the path of Euler (1707-1783) and Lagrange, Jacobi (1804-1851) and Hamilton (1805-1865) continued the development of analytic techniques for the explicit solution of the differential equations describing mechanical systems. Finally, geometry has taken a new role in mechanics through the contributions of Poincaré (1854-1912) and Birkhoff (1884-1944). Now, though, the geometry is the more flexible geometry of canonical (in particular, area preserving) transformations instead of the rigid geometry of Euclid; accordingly, the conclusions of the geometrical arguments are often qualitative rather than quantitative.

In this lecture (and paper), I would like to explain what symplectic geometry is and to describe its role in contemporary mathematics. I think it is not unfair to say that symplectic geometry is of interest today, not so much as a theory in itself, but rather because of a series of remarkable "transforms" which connect it with various areas of analysis. ${ }^{2}$

The lagrangian submanifolds play an especially important part in symplectic geometry and its applications. In $\$ 3$ of this lecture, I will outline an approach to symplectic geometry in terms of a "category" in which the morphisms are exactly the lagrangian submanifolds; this approach suggests some interesting

Presented at the Symposium on the Mathematical Heritage of Henri Poincare in April, 1980; received by the editors November 15, 1980.

1980 Mathematics Subject Classification. Primary 58F05.

${ }^{1}$ Poinsot (1777-1859) reacted strongly against this analytical tradition. Referring to "l'illustre Lagrange" in his famous study of rigid body rotation, Poinsot wrote that in Lagrange's treatment of the subject, "on ne voit guère que des calculs, sans aucune image nette de la rotation du corps." (I would like to thank J. J. Duistermaat for calling my attention to Poinsot's vivid critique of analytical mechanics, as well as for his comments on this manuscript.)

2 G. D. Birkhoff's “disturbing secret fear that geometry may ultimately turn out to be no more than the glittering intuitional trappings of analysis" [BI] may be especially appropriate when applied to symplectic geometry. I learned of Birkhoff's statement from Chern [C], who tends to dismiss the fear on the grounds that Birkhoff was an analyst. The recent success of symplectic geometric methods in linear partial differential equations (see $\$ 5$ for an example) suggests that one might need the glitter to find the gold. This opinion is also expressed by Poinsot (see previous footnote) who suggests that calculations are merely a tool in the service of geometrical and mechanical reasoning. 
new problems as well as unifying previous results. Some of the ideas in this section were developed independently by Guillemin and Sternberg, implicitly in [G-S1] and explicitly in $\$ \$ 9$ and 10 of [G-S2].

1. Symplectic spaces and manifolds. The model space for symplectic geometry is the $2 n$-dimensional cartesian space $\mathbf{R}^{2 n}$ with symplectic structure provided by the differential form $\Omega_{n}=\sum_{j=1}^{n} d q_{i} \wedge d p_{i}$ in coordinates $\left(q_{1}, \ldots, q_{n}, p_{1}, \ldots, p_{n}\right)$. Eventually, we shall consider differentiable transformations preserving this structure, but for a moment let us look at the linear ones, from which the name "symplectic" comes.

The planes (through the origin) in $\mathbf{R}^{2 n}$ on which $\Omega_{n}$ vanishes (as a bilinear form) form a hypersurface $C$ in the Grassmann manifold $G_{2,2 n}(\mathbf{R})$. The Grassmann manifold may be identified with the space of lines in $\mathbf{R} P^{2 n-1}$, and a hypersurface in this space of lines is called a line complex in projective geometry. These complexes were studied extensively by Plücker (1801-1868) and others in the 19th century. The complex $C$ is called a linear complex, since it is defined by linear equations on the exterior product $\mathbf{R}^{2 n} \wedge \mathbf{R}^{2 n}$, so the group of projective transformations leaving $C$ invariant (equal to the projectivization of the linear group leaving $\Omega_{n}$ invariant) was called the linear complex group. In 1946, Hermann Weyl [WL] decided that the terminology was too confusing to perpetuate, so he took the Latin roots in com-plex (meaning "plaited together") and replaced them by the Greek roots symplectic. $^{3}$ The name symplectic group is now universally used for the group of linear transformations preserving $\Omega_{n}$.

The mathematical point to be retained from this linguistic digression is that the symplectic structure is essentially determined by the subspaces on which it vanishes. In addition to the planes in $\mathbf{R}^{2 n}$ on which $\Omega_{n}$ vanishes, we may consider larger subspaces with this property, called isotropic. The maximal isotropic subspaces have dimension $n$ and are called lagrangian (following Maslov [MAS]).

A submanifold $L$ of $\mathbf{R}^{2 n}$ is called lagrangian if its tangent space at each point is lagrangian, i.e. if $L$ is $n$-dimensional, and the pullback of $\Omega_{n}$ to $L$ vanishes. If $L$ projects diffeomorphically onto the subspace $\left\{\left(q_{1}, \ldots, q_{n}, 0, \ldots, 0\right)\right\}$, so that $L$ is defined by equations of the form $p_{i}=\theta_{i}\left(q_{i}, \ldots, q_{n}\right)$, then it is easy to verify that $L$ is lagrangian if and only if $\partial \theta_{i} / \partial q_{j}=\partial \theta_{j} / \partial q_{i}$ on $\mathbf{R}^{n}$, i.e. if and only if there is a function $S\left(q_{1}, \ldots, q_{n}\right)$ such that $\theta_{i}=\partial S / \partial q_{i}$. Such a function $S$ is called a generating function for the lagrangian submanifold $L$. One of the most important recent developments in the applications of symplectic geometry is the discovery that, if we identify this $L$ with the function $e^{i S}$, then we can extend this identification so that more general lagrangian submanifolds (not necessarily projecting diffeomorphically onto $\mathbf{R}^{2 n}$ ) correspond to generalized functions on $\mathbf{R}^{n}$. (See [G-S1] for a detailed account of this correspondence.) In a sense, we may think of the

\footnotetext{
${ }^{3}$ According to the Oxford English Dictionary, the only use of "symplectic" in English is with reference to a certain bone in the head of fish. The dictionary gives a citation from Todd's Cyclopedia of Anatomy (1839-47). A picture of the symplectic bone may be found on p. 67 of [BO]; it appears that the bone is quite small, but that it may serve to hold things together.
} 
lagrangian submanifolds themselves as being a generalization of functions.

The symplectic group is too rigid for many purposes. The class of lagrangian submanifolds, for instance, is invariant under the group of all diffeomorphisms $f: \mathbf{R}^{2 n} \rightarrow \mathbf{R}^{2 n}$ for which $f^{*} \Omega_{n}=\Omega_{n}$. More generally, we may consider diffeomorphisms between open subsets of $\mathbf{R}^{2 n}$ which preserve $\Omega_{n}$; these diffeomorphisms are called canonical transformations, symplectic diffeomorphisms, or (following Souriau [SO]), symplectomorphisms. We may define a symplectic manifold to be a differentiable manifold with an atlas for which the coordinate changes are symplectomorphisms. Alternately, we may define a symplectic structure on a manifold $P$ as a closed 2 -form $\Omega$ which is nondegenerate in the sense that the bundle map $\tilde{\Omega}: T P \rightarrow T^{*} P$ defined by $[\tilde{\Omega}(v)](w)=\Omega(v, w)$ is an isomorphism. It is a theorem of Darboux that, near any point in a manifold with a symplectic structure, there are local coordinates $\left(q_{1}, \ldots, q_{n}, p_{1}, \ldots, p_{n}\right)$ for which $\Omega=\Sigma_{i} d q_{i} \wedge d p_{i}$, so the two definitions are equivalent.

An important class of symplectic manifolds consists of the cotangent bundles $T^{*} X$. Every local coordinate system $\left(q_{1}, \ldots, q_{n}\right)$ on $X$ gives rise to local coordinates $\left(q_{1}, \ldots, q_{n}, p_{1}, \ldots, p_{n}\right)$ on $T^{*} X$, and the form $\Sigma d q_{1} \wedge d p_{i}$ is independent of the choice of such coordinates; we denote the symplectic structure on $T^{*} X$ by $\Omega_{X}$. The image of a section $\sigma: X \rightarrow T^{*} X$ is a lagrangian submanifold of $\left(T^{*} X, \Omega_{X}\right)$ if and only if $d \sigma=0$; if $\sigma=d S, S$ is called a generating function for $\sigma(X)$.

2. Symplectic geometry as Lagrange did it. The first symplectic manifold was introduced by Lagrange [LA1] in $1808 .^{4}$ In studying the motion of the planets under the influence of their mutual gravitational interaction, he took as a starting point the elliptical motion of a single planet around the sun. (Actually, the focus of the ellipse is the center of the mass of the sun-planet system, but we will ignore this point here.) This ellipse was then considered to "drift" under the disturbing influence of the other planets. The drift was described by a system of differential equations, and Lagrange sought to put these equations in the simplest possible form.

Let $\mathcal{E}$ be the set of possible elliptical motions of a planet. A member of $\mathcal{E}$ may be described by six real numbers, called elements of the orbital motion. For instance, one may take the major axis and eccentricity of the ellipse, two variables describing the orientation of the plane containing the ellipse, one variable giving the orientation of the ellipse within this plane, and a final variable specifying the time at which the planet passes a designated point on the ellipse. In modern terms, we may say that $\mathcal{E}$ is a six-dimensional manifold, with the elements forming a local coordinate system. (Chapter 9 of [A-M] contains a nice discussion of various systems of elements.)

Let $a_{1}, \ldots, a_{6}$ be a system of elements. The problem faced by Lagrange and his contemporaries, and solved by them in many different ways, was to write down a system of first-order differential equations for the $a_{j}$ 's. Finally, in 1808, Lagrange came up with a formulation of these equations which was

\footnotetext{
${ }^{4}$ In this section, we take the symposium title "Mathematical Heritage of Poincare" to refer to what Poincaré inherited, rather than what he left for us.
} 
much simpler than any which had been found before. He first constructed, for any system of elements, and each pair of indices with $1<i, j<6$, a "bracket" $\left[a_{i}, a_{j}\right]$ which, like each of the $a_{i}$ 's, is a function on $\mathcal{E}$. The bracket expressions (now called Lagrange brackets) satisfy the antisymmetry condition

$$
\left[a_{i}, a_{j}\right]=-\left[a_{j}, a_{i}\right] \text {. }
$$

Lagrange also constructed a real valued function $\Phi$ on $\mathcal{E}$, called the disturbing function, which depends on the perturbing forces but is independent of the choice of elements, such that the drift caused by the perturbation satisfies the equations:

$$
\frac{\partial \Phi}{\partial a_{i}}=\sum_{j=1}^{6}\left[a_{i}, a_{j}\right] \frac{d a_{j}}{d t} .
$$

The matrix of brackets satisfies the nondegeneracy condition

$$
\operatorname{det}\left(\left[a_{i}, a_{j}\right]\right) \neq 0
$$

so there is an inverse matrix $\left(b_{i j}\right)$, and the equations (2) become

$$
\frac{d a_{i}}{d t}=\sum_{j=1}^{6} b_{i j} \frac{\partial \Phi}{\partial a_{j}}
$$

in which the derivatives of the elements are expressed in terms of one function instead of six. At the time of Lagrange, this advance was of advantage largely because it shortened by a factor of six some rather complicated computations. The theoretical properties of equations of the form (4) were yet to be explored, beyond the following further results due to Lagrange.

The antisymmetry of the matrix $\left(b_{i j}\right)$ implies that the total derivative of $\Phi$ with respect of time along a solution of (4) is zero; i.e. the disturbing function $\Phi$ is a conserved quantity for the drift motion. This conservation law could be used to check computations, as well as in discussions of stability.

Lagrange also found another invariant for the solutions of (4), which we may describe as follows. Let $f_{t}: \mathcal{E} \rightarrow \mathcal{E}$ be the mapping which associates to each elliptical orbit $e$ the elliptical orbit $f_{t}(e)$ to which the planet will have drifted from $e$ after the perturbing forces have acted for $t$ units of time. For any value of $t$, the six functions $a_{i} \circ f_{t}$ are a new set of elements, and the brackets $\left[a_{i} \circ f_{t}, a_{j} \circ f_{t}\right]$ may be formed. Lagrange's last invariance principle states that the functional form of the expression of $\left[a_{i} \circ f_{t}, a_{j} \circ f_{t}\right]$ in terms of the $a_{i} \circ f_{t}$ 's is independent of time, i.e.

$$
\left[a_{i} \circ f_{t}, a_{j} \circ f_{t}\right]=\left[a_{i} \circ a_{j}\right] \circ f_{t} .
$$

Finally, in [LA2], Lagrange found special elements $\left(q_{1}, q_{2}, q_{3}, p_{1}, p_{2}, p_{3}\right)$ for which $\left[q_{i}, q_{i}\right]=\left[p_{i}, p_{j}\right]=0$ and $\left[p_{i}, q_{j}\right]=\delta_{i j}$. In terms of these elements, the equations (4) become

$$
d q_{i} / d t=\partial \Phi / \partial p_{i}, \quad d p_{i} / d t=-\partial \Phi / \partial q_{i}
$$

which are known today as Hamilton's equations.

In modern differential geometric terms, we may identify the Lagrange brackets $\left[a_{i}, a_{j}\right]$ as the coefficients $\Omega\left(\partial / \partial a_{j}, \partial / \partial a_{i}\right)$ of a differential 2 -form $\Omega$. 
Condition (1) means that $\Omega$ is a skew-symmetric form, while condition (3) means that $\Omega$ is nondegenerate in the sense defined in $\S 1$. Equation (2) is now expressed in the form $d \Phi=\tilde{\Omega} \circ \xi$, where $\xi$ is the vector field describing the drift motion, and equation (4) becomes $\xi=\tilde{\Omega}^{-1} \circ d \Phi$. With $f_{t}$ defined as the map at time $t$ of the flow generated by $\xi$, equation (5) states that $f_{t}^{*} \Omega=\Omega$, i.e. $\Omega$ is invariant under the flow. It turns out that this invariance condition (for all functions $\Phi$ ) is equivalent to the form $\Omega$ being closed, i.e. $d \Omega=0$. Note that if $\Sigma$ is any 2-cycle in $\mathcal{E}$, then $\int_{\Sigma} \Omega=\int_{f_{t}(\Sigma)} \Omega$. The invariance principle in this form was discovered by Poincaré, who called $\Omega$ an integral invariant. Finally, in terms of the special elements $\left(q_{1}, q_{2}, q_{3}, p_{1}, p_{2}, p_{3}\right), \Omega$ is equal to $\Sigma_{i} d q_{i} \wedge d p_{i}$, as in the conclusion of Darboux's theorem.

Since the time of Lagrange, the general form (4) of the equations of drift (or motion) seems largely to have been abandoned in favor of the "canonical" form (6). Recently, inspired by the geometric point of view, R. Littlejohn [LI] has found that the use of (6) is well suited to some computations concerning the stability of motion of a charged particle in a magnetic field, a problem of interest in the physics of accelerators, plasmas, and the earth's atmosphere. This application of geometrically based ideas to a concrete physical problem is very much in the spirit of Poincaré's work in classical mechanics.

3. The symplectic "category". The constructions described in the last section can be carried out on any symplectic manifold $(P, \Omega)$. If $\xi$ is a vector field on $P$, then the flow of $\xi$ leaves $\Omega$ invariant if and only if $\tilde{\Omega} \circ \xi$ is closed 1-form. As we saw in $\S 1,(\tilde{\Omega} \circ \xi)(P)$ is a lagrangian submanifold of $T^{*} P$, so we have a correspondence between infinitesimal symplectomorphisms of $\boldsymbol{P}$ and certain lagrangian submanifolds of $T^{*} P$. The correspondence is an illustration of what I might call the "symplectic creed":

\section{EVERYTHING IS A LAGRANGIAN SUBMANIFOLD}

In practice, the symplectic creed means that one should try to express objects and constructions in symplectic geometry in terms of lagrangian submanifolds. This approach, which lends a certain unity to symplectic geometry, is particularly fruitful in view of the transforms of Hörmander [HR], Maslov [MAS], and Sato [S-K-K] which assign classes of generalized functions to lagrangian submanifolds of cotangent bundles.

After the images of closed 1 -forms, the next most important lagrangian submanifolds are the graphs of symplectomorphisms. If $\left(P_{1}, \Omega_{1}\right)$ and $\left(P_{2}, \Omega_{2}\right)$ are symplectic manifolds, the product $P_{1} \times P_{2}$ may be given the symplectic structure $\pi_{1}^{*} \Omega_{1}-\pi_{2}^{*} \Omega_{2}$, where $\pi_{j}: P_{1} \times P_{2} \rightarrow P_{j}$ are the cartesian projections. Now a diffeomorphism $f: P_{2} \rightarrow P_{1}$ is a symplectomorphism if and only if its graph is lagrangian in $\left(P_{1} \times P_{2}, \pi_{1}^{*} \Omega_{1}-\pi_{2}^{*} \Omega_{2}\right)$. Since a symplectomorphism is also called a canonical transformation, and an arbitrary subset of $P_{1} \times P_{2}$ is a relation, the lagrangian submanifolds of $\left(P_{1} \times P_{2}, \pi_{1}^{*} \Omega_{1}-\pi_{2}^{*} \Omega_{2}\right)$ have been named by Hörmander [HR] canonical relations from $P_{2}$ to $P_{1}$. (They were also studied by Sniatycki and Tulczejew [SN-T], who called them symplectic relations.) It was proven by Hörmander that the set-theoretic composition of canonical relations is again a canonical relation, provided that 
a transversality condition is satisfied. (A weaker form of the transversality condition was found to be sufficient by Guillemin [G] and the author [WE1].)

To systematize the application of the symplectic creed, we may define the symplectic "category" $\mathfrak{S}$, in which the objects are symplectic manifolds, the morphisms are canonical relations, and composition of morphisms is set-theoretic composition of relations. $\mathcal{S}$ is not a true category, since not all compositions are morphisms in $\mathcal{\delta}$, but we can still use the language of category theory to study canonical relations from this point of view. ${ }^{5}$

A point in a category is an object which has only one endomorphism; in $\mathfrak{S}$, any point is isomorphic to the zero-dimensional manifold $\mathbf{R}^{\mathbf{0}}$; the only $\mathbf{2}$-form on $\mathbf{R}^{0}$ is 0 , but this is a symplectic structure. Now we may define the elements of an object in a category to be the morphisms from a point to the object. In $\mathcal{\delta}$, the elements of $(P, \Omega)$ are the lagrangian submanifolds of $\left(P \times \mathbf{R}^{0}, \pi_{1}^{*} \Omega-\right.$ $\pi_{2}^{* 0} 0$, which is naturally isomorphic to $(P, \Omega)$; i.e. the elements of $(P, \Omega)$ are its lagrangian submanifolds.

The symplectic manifold $\left(P_{1} \times P_{2}, \pi_{1}^{*} \Omega_{1}+\Omega_{2}^{*} \pi_{2}\right)$ plays the role in $\delta$ of the product $\left(P_{1}, \Omega_{1}\right) \times\left(P_{2}, \Omega_{2}\right)$; a pair of morphisms from $\left(P_{j}^{\prime}, \Omega_{j}^{\prime}\right)$ to $\left(P_{j}, \Omega_{j}\right)$ $(j=1,2)$ gives rise to a morphism from $\left(P_{1}^{\prime}, \Omega_{1}^{\prime}\right) \times\left(P_{2}^{\prime}, \Omega_{2}^{\prime}\right) \rightarrow\left(P_{1}, \Omega_{1}\right) \times$ $\left(P_{2}, \Omega_{2}\right)$ (note that the projections are not in $\mathcal{S}$ ). The disjoint union of $\left(P_{1}, \Omega_{1}\right)$ and $\left(P_{2}, \Omega_{2}\right)$ acts as a sum.

A reflexive duality in $\mathcal{S}$ is given by the contravariant functor which assigns to each $(P, \Omega)$ the manifold $(P, \Omega)^{*}=(P,-\Omega)$ and to each canonical relation its inverse. Notice that the morphisms from $\left(P_{2}, \Omega_{2}\right)$ to $\left(P_{1}, \Omega_{1}\right)$ are precisely the elements of $\left(P_{1}, \Omega_{1}\right) \times\left(P_{2}, \Omega_{2}\right)^{*}$; it is reasonable, therefore, to denote the latter product by $\operatorname{Hom}\left(\left(P_{2}, \Omega_{2}\right),\left(P_{1}, \Omega_{1}\right)\right)$. (Compare this with the isomorphism $\operatorname{Hom}\left(V_{2}, V_{1}\right) \simeq V_{1} \otimes V_{2}^{*}$ in the category of vector spaces.)

For any object $(P, \Omega)$ in $\mathcal{S}$, the object $\operatorname{Hom}((P, \Omega),(P, \Omega))$, which we denote by $\operatorname{End}(P, \Omega)$, has the structure of a "*-algebra" in the category $\delta$. Namely, we have:

(i) A multiplication morphism from $\operatorname{End}(P, \Omega) \times \operatorname{End}(P, \Omega)$ to $\operatorname{End}(P, \Omega)$, i.e. from $(P, \Omega) \times(P, \Omega)^{*} \times(P, \Omega) \times(P, \Omega)^{*}$ to $(P, \Omega) \times(P, \Omega)^{*}$, given by

$$
\left\{\left(p_{1}, p_{2}, p_{3}, p_{4}, p_{5}, p_{6}\right) \in P^{6} \mid p_{1}=p_{3}, p_{2}=p_{6}, p_{4}=p_{5}\right\}
$$

(ii) An identity element of $\operatorname{End}(P, \Omega)$ given by the diagonal in $P \times P$;

(iii) An involution from $\operatorname{End}(P, \Omega)$ to its dual given by

$$
\left\{\left(p_{1}, p_{2}, p_{3}, p_{4}\right) \mid p_{1}=p_{4}, p_{2}=p_{3}\right\} \text {. }
$$

Another important example of a ${ }^{*}$-algebra in $\delta$ is given by $\left(T^{*} G, \Omega_{G}\right)$, where $G$ is a Lie group; the operations are:

(i) the multiplication morphism

$$
M \subseteq\left(T^{*} G, \Omega_{G}\right) \times\left(T^{*} G,-\Omega_{G}\right) \times\left(T^{*} G,-\Omega_{G}\right)
$$

given by $\left\{\left(\left(g_{1}, \gamma_{1}\right),\left(g_{2}, \gamma_{2}\right),\left(g_{3}, \gamma_{3}\right)\right) \mid \gamma_{j} \in T_{g_{j}}^{*} G, g_{1}=g_{2} g_{3}, \gamma_{2}=r_{g_{3}}^{*} \gamma_{1}, \gamma_{3}=\right.$ $\left.l_{g_{2}}^{*} \gamma_{1}\right\}$, where $l_{g}^{*}$ and $r_{g}^{*}$ are the pullbacks by left and right translation by $g$ in

\footnotetext{
5 A subcategory of $\mathcal{S}$, consisting of linear symplectic spaces and lagrangian subspaces, was introduced (and quantized) by Guillemin and Sternberg in [G-S2]. Thus, they must have had the idea of $\mathcal{S}$ in mind, although they did not develop it in the way described here.
} 
G. $M$ may also be described as the normal bundle in $T^{*}(G \times G \times G)$ of the graph $\left\{\left(g_{1}, g_{2}, g_{3}\right) \mid g_{1}=g_{2} g_{3}\right\} \subseteq G \times G \times G$, with the sign of the last two covectors reversed; analytically, $M$ is the wavefront set (see [HR]) of the convolution operator on the group algebra of $G$.

(ii) The identity element given by the cotangent space at the identity $T_{e}^{*} G \subseteq T^{*} G$.

(iii) The involution from $\left(T^{*} G, \Omega_{G}\right)$ to its dual given by $\left\{\left(\left(g_{1}, \gamma_{1}\right)\right.\right.$, $\left.\left.\left(g_{2}, \gamma_{2}\right)\right) \mid g_{1}=g_{2}^{-1}, \gamma_{1}=-\mathrm{inv}^{*} \gamma_{2}\right\}$, where inv: $G \rightarrow G$ is the inversion mapping.

For every manifold $X$, the symplectic manifold $\left(T^{*} X, \Omega_{X}\right)$ is a commutative *-algebra in $\mathcal{S}$, the operations being:

(i) The multiplication morphism $\left\{\left(x_{1}, \xi_{1}\right),\left(x_{2}, \xi_{2}\right),\left(x_{3}, \xi_{3}\right) \mid x_{1}=x_{2}=x_{3}\right.$, $\left.\xi_{1}=\xi_{2}+\xi_{3}\right\}$, which is the wavefront set of the ordinary multiplication operator for functions on $X$.

(ii) The identity element given by the zero section in $T^{*} X$.

(iii) The involution from $\left(T^{*} X, \Omega_{X}\right)$ to its dual given by $\left\{\left(\left(x_{1}, \xi_{1}\right)\right.\right.$, $\left.\left.\left(x_{2}, \xi_{2}\right)\right) \mid x_{1}=x_{2}, \xi_{1}=-\xi_{2}\right\}$.

Now we may consider homomorphisms between *-algebras in $\mathcal{S}$, i.e. morphisms which are compatible with the three parts of the *-algebra structure. A simple example is given by the "Fourier transform", as follows. Let $G=X=\mathbf{R}^{n}$, considered first as a Lie group and then as a manifold, so that $T^{*} \mathbf{R}^{n}$ is a ${ }^{*}$-algebra in two different ways. A homomorphism from the "convolution" to the "multiplication" structure is given by

$$
\begin{aligned}
& \mathscr{B}=\left\{((x, \xi),(g, \gamma)) \mid x \in \mathbf{R}^{n}, g \in \mathbf{R}^{n}, \xi \in\left(\mathbf{R}^{n}\right)^{*}, \gamma \in\left(\mathbf{R}^{n}\right)^{*},\right. \\
& \gamma=B x, \xi=-B g\}
\end{aligned}
$$

where $B: \mathbf{R}^{n} \rightarrow \mathbf{R}^{n^{*}}$ is any symmetric linear transformation. ( $B$ need not be invertible for $\mathscr{B}$ to be a canonical relation, but only for $B$ invertible is $\mathscr{B}$ a homomorphism from the multiplication to the convolution structure.)

Another important class of homomorphisms in $\mathfrak{S}$ arises from actions of Lie groups on symplectic manifolds. Whenever a Lie group $G$ acts differentiably by symplectomorphisms on a symplectic manifold $(P, \Omega)$, there is an induced Lie algebra homomorphism $\alpha$ from the Lie algebra $g$ of $G$ to the Lie algebra $\Xi(P, \Omega)$ of infinitesimal symplectomorphisms of $(P, \Omega)$. It is known that, for purposes of quantization, some extra structure should be brought in; namely, the homomorphism $\alpha$ should be lifted to a homomorphism $\beta: \mathfrak{g} \rightarrow C^{\infty}(P, \Omega)$; here, $C^{\infty}(P, \Omega)$ has the Lie algebra structure given by the Poisson bracket operation $\left\{\Phi_{1}, \Phi_{2}\right\}=\Omega\left(\xi_{\Phi_{1}}, \xi_{\Phi_{2}}\right)$, where $\xi_{\Phi}=\tilde{\Omega}^{-1} \circ d \Phi$. (The Jacobi identity for this operation is equivalent to the equation $d \Omega=0$.) Another way to look at the homomorphism $\beta$ is as a map $\mu: P \rightarrow \mathrm{g}^{*}$ defined by $[\mu(p)](v)=$ $[\beta(v)](p)$, for $p \in P$ and $v \in \mathfrak{g}$. If $G$ is connected, the map $\mu$, called a momentum map for the action, is equivariant with respect to the coadjoint representation of $G$ on $\mathrm{g}^{*}$; for general $G$, this equivariance must be taken as an additional condition.

All this extra structure associated with a group action becomes quite natural from the point of view of the symplectic category. Given an action of 
$G$ on $P$ and a mapping $\mu: P \rightarrow \mathrm{g}^{*}$, we may define a mapping $A: P \times G \rightarrow$ $P \times P \times T^{*} G$ by the formula

$$
A(p, g)=\left(g p, p, l_{g^{-1}} \mu(p)\right)
$$

It turns out (see p. 21 of [WE3], or [WE4]) that $L=A(P \times G)$ is a homomorphism from $\left(T^{*} G, \Omega_{G}\right)$ to $\operatorname{End}(P, \Omega)$ if and only if $\mu$ is an equivariant momentum mapping coming from a Lie algebra homomorphism $\mathrm{g} \rightarrow$ $C^{\infty}(P, \Omega)$; the action of $G$ on $P$, as well as $\mu$, can then be recovered from $L$. In terms of $\mathcal{S}$, therefore, $L$ is the action; it is an action of the symplectic *-algebra $T^{*} G$ on $P$ and contains all the extra structure which is usually obtained from quantization theory.

4. Quantization. The mathematical quantization problem arises from the physical problem of assigning quantum-mechanical observables (self-adjoint operators on a Hilbert space) to corresponding classical-mechanical ones (real valued functions on $\mathbf{R}^{2 n}$ ). If one requires that Poisson brackets go over to (a multiple of) commutator brackets, then the quantization problem has no solution. (Van Hove's theorem; see [A-M].) One way to make progress on the quantization problem is to make it simultaneously harder and easier. We enlarge the class of classical objects which are to be quantized, but we relax the requirements by which the quantum and classical objects are to correspond.

The first step in enlarging the problem is to replace the observables by the groups of which they are the infinitesimal generators. Our basic classical objects are then symplectomorphisms, which should be represented by unitary operators on quantum Hilbert spaces. This idea was introduced already by Weyl and von Neumann as a way of avoiding some of the analytical difficulties connected with unbounded operators.

The category $\mathfrak{S}$ suggests an approach to the quantization problem by a still further extension. First of all, to every symplectic manifold $(P, \Omega)$ we try to assign a Hilbert space $H(P, \Omega)$. This should be done in such a way that $H(P,-\Omega)$ is the dual of $H(P, \Omega)$, and $H\left[\left(P_{1}, \Omega_{1}\right) \times\left(P_{2}, \Omega_{2}\right)\right]$ is a tensor product of $H\left(P_{1}, \Omega_{1}\right)$ and $H\left(P_{2}, \Omega_{2}\right)$. Now, for every lagrangian submanifold $L \subseteq(P, \Omega)$, we should try to assign an element of $H(P, \Omega)$. In particular, for every morphism in $\mathcal{S}$ from $\left(P_{2}, \Omega_{2}\right)$ to $\left(P_{1}, \Omega_{1}\right)$, we will get an operator from $H\left(P_{2}, \Omega_{2}\right)$ to $H\left(P_{1}, \Omega_{1}\right)$. Simple examples show that it may take more data than just $L$ to specify an element of $H$; furthermore, the analytic object constructed from $L$ may be in some extension of $H$ (e.g. a distribution rather than an $L^{2}$ function). Nevertheless, the general idea outlined here has proven extremely fruitful when complemented by hard analysis in specific situations.

An excellent example of the quantization procedure just outlined is the theory of Fourier integral operators. (See the lecture of Leray in this symposium for another example.) Here the symplectic manifolds considered are all obtained from cotangent bundles with their zero sections deleted by taking duals and products; the Hilbert space assigned to $\left(\dot{T} X, \Omega_{X}\right)$ is the space $|X|^{1 / 2}$ of $L^{2}$ half-densities on $X$; the lagrangian submanifolds quantized are those which are invariant under multiplication by real positive scalars. If $\Gamma_{f}$ is the 
graph of a homogeneous symplectomorphism $f: \dot{T}^{*} X \rightarrow \dot{T}^{*} Y$, then $\Gamma_{f}$ is quantized by a whole class $C_{f}$ of operators from $|X|^{1 / 2}$ to $|Y|^{1 / 2}$. Barring a possible obstruction of Fredholm index type (see [WE2]) one can choose a unitary operator $U_{f}$ in $C_{f}$. Ideally, one would like to choose the $U_{f}$ 's such that $U_{f g}$ is always equal to $U_{f} U_{g}$, but such a choice is probably impossible. (A rigorous proof of this impossibility may not have been published anywhere, but the ideas behind van Hove's theorem should apply.) Instead, one finds that $U_{f g}-U_{f} U_{g}$ is generally a nonzero pseudodifferential operator of order -1 . It is unknown to what extent (for specific sets or groups of $f$ 's) the error terms $U_{f g}-U_{f} U_{g}$ can be made to have lower order, or be smoothing operators, or be zero. One result in the positive direction is that any compact group of homogeneous symplectomorphisms can be represented homomorphically by unitary Fourier integral operators. This was proven by the author (unpublished) by interpreting the operator-theoretic constructions of $[\mathbf{H}-\mathbf{K}]$ in terms of $\mathcal{S}$, and by Boutet de Monvel with the aid of his abstract Toeplitz structures [BU].

The theory of Fourier integral operators illustrates an important feature of quantization, which is also stated quite explicitly by Maslov [MAS]. The Hilbert spaces associated to symplectic manifolds usually carry some sort of filtration, and the quantization is only "correct" to within a certain degree of accuracy as measured by the filtrations. It is only in special situations that approximate or asymptotic correspondences can be made into exact ones, and in those cases there are usually some ideas from outside of symplectic geometry which are brought into play (e.g. representation theory in [AU-K], and the theory of hyperbolic equations in [D-H]).

To close this section, we shall briefly describe the "geometric quantization" scheme of Kostant [KO1] and Souriau [SO], motivated by earlier work of Segal [SE] and Kirillov [KI1]. To quantize a symplectic manifold $(P, \Omega)$ by this scheme, one begins by finding a principal circle bundle $Q \rightarrow P$ whose curvature form is $\Omega$. One then defines $H(P, \Omega)$ to be, roughly, a space of sections of the associated complex line bundle which are covariant constant along the leaves of a foliation by lagrangian manifolds; such a foliation is called a polarization. We highly recommend the recent monograph by Sniatycki [SN] for a thorough discussion of this quantization theory.

The category $\delta$ might prove useful in geometric quantization; here are a few suggestions. First of all, the circle bundle and polarization constructions can be interpreted in $\mathcal{S}$. Instead of looking at the space $Q$, which with the connection form $\phi$ is a contact manifold, we may consider its "symplectification" $\hat{Q}=\left\{r \phi(q) \in T^{*} Q \mid q \in 0, r>0\right\}$ with the symplectic structure induced by the embedding in $\left(T^{*} Q, \Omega_{Q}\right)$. There is a natural action of the symplectic algebra $T^{*} S^{1}$ on $Q$, coming from a symplectic action of $S^{1}$ on $Q$, and one of the corresponding reduced manifolds (see [AR], [MA-W], or [MEY] for the theory of reduction) is $(P, \Omega)$. The other reduced manifolds are the manifolds $(P, r \Omega)$ for $r>0$; since one can now let $r \rightarrow \infty$, the circle bundle construction introduces asymptotics into the situation. (Some of the ideas expressed here are borrowed from Boutet de Monvel and Guillemin [BU-G], who develop a quantization procedure for contact manifolds which 
leads to a remarkable unification of spectral theory with the theory of the Hilbert polynomial in algebraic geometry.)

Polarizations can be interpreted in $\mathcal{S}$, too. If $\mathcal{E}$ is a foliation of $(P, \Omega)$ by lagrangian submanifolds, we consider the submanifold $C_{\mathfrak{L}} \subseteq P \times P$ defined as $\left\{\left(p_{1}, p_{2}\right) \mid p_{1}\right.$ and $p_{2}$ are in the same leaf of $\left.\mathcal{L}\right\}$. This is an immersed coisotropic submanifold of $\operatorname{End}(P, \Omega)$. Now every coisotropic submanifold $C$ of a symplectic manifold $\left(P_{1}, \Omega_{1}\right)$ carries the characteristic foliation by lagrangian submanifolds, and we may define an immersed lagrangian submanifold $L(C) \subseteq \operatorname{End}\left(P_{1}, \Omega_{1}\right)$ by

$$
\begin{aligned}
L(C)=\{( & \left(c_{1}, c_{2}\right) \mid c_{1} \in C, c_{2} \in C, \\
& \left.c_{1} \text { and } c_{2} \text { are in the same leaf of the characteristic foliation }\right\} .
\end{aligned}
$$

$L(C)$, considered as a morphism from $\operatorname{End}\left(P_{1}, \Omega_{1}\right)$ to itself, is idempotent, so it is like a projection operator in $\mathcal{S}$. (This fact may be "quantized" in Fourier integral operator theory; see [G-S2]. $L(C)$ has also been used in [MO].) Putting together the two observations above, we find that a polarization of $(P, \Omega)$ leads to a "projection operator" on $\operatorname{End}(P, \Omega)$ and thus corresponds to a subspace (subalgebra?) of "operators" on $(P, \Omega)$.

Finally, we propose the following synthesis of geometric quantization and Fourier integral operator theory. Given a symplectic manifold $(P, \Omega)$ together with circle bundle and polarization, and a lagrangian submanifold $L \subseteq$ $(P, \Omega)$, construct an element or class of elements of $H(P, \Omega)$. Use of the fact, clear from Fourier integral operator theory, that some extra data besides $L$ may be necessary might help to resolve some of the nonunitarity problems described in [G-S1] and [SN].

5. Some applications of symplectic geometry. In this section, I shall describe very briefly a (by no means complete) sample of the recent applications of symplectic geometry. In each case, the application begins with a "transform" which brings symplectic geometry into the area of interest.

A. Conservative mechanics. The law of motion of a system of bodies is commonly given as a system of first order differential equations in the positions and velocities of the bodies. The Legendre transform replaces the velocities by momenta and puts the equations in hamiltonian form. Methods of symplectic geometry such as the Birkhoff normal form, the KolmogorovArnold-Moser theorem on the persistence of invariant tori, the intersection theory of lagrangian submanifolds, and the classification of group actions on symplectic manifolds may now be applied to study questions of stability, periodicity, and symmetry in conservative mechanics. (A good general reference in this area is [AR]. Also see [A-M], [G-S1], [SO], and [ST].)

B. Completely integrable systems. The nice structure of the solutions of the Korteweg-de Vries partial differential equation $u_{t}=-u_{x x x}+6 u u_{x}$ originally discovered by Gardner, Green, Kruskal and Miura is now explained neatly in terms of the Faddeev-Zaharov symplectic structure [F-Z] on the space of functions on $\mathbf{R}$, which makes the $K d V$ equation into an infinite-dimensional hamiltonian system. A closely related finite dimensional system is the Toda lattice; this example has been studied and generalized by, among many 
others, Kostant [KO2], who uses as a basic tool the technique of reduction of symplectic manifolds with symmetry (see [K-K-S], [M], [MA-W], [MEY]). Another application of reduction to completely integrable systems is given by Mishchenko and Fomenko in [MI-F].

C. Representation theory. The geometric quantization scheme of Segal-Kirillov-Kostant-Souriau (see §4) leads to a correspondence between irreducible unitary representations of a Lie group $G$ and transitive symplectic actions of these groups. The correspondence is essentially bijective for nilpotent, compact, and certain solvable groups. Although there are examples where the correspondence fails (see [R-W]), the symplectic viewpoint, often called the orbit method, since the homogeneous symplectic manifolds are coadjoint orbits, is at least a useful heuristic tool for investigating the representations of more general groups. The most extensive discussion available of the orbit method can be found in [KI2]. The nilpotent case is also treated in detail in [WA]. The coadjoint orbits have also appeared recently in the work of Simon [SI] on the statistical mechanics of representations of compact groups.

D. Linear partial differential equations. The relevant "transforms" here are the Fourier integral operator calculus of Hörmander [HR], the canonical operator of Maslov [MAS], and the quantized contact transformation theory of Sato [S-K-K]. In each case, when a differential operator on a manifold $X$ is represented by a function on $T^{*} X$ (its principal symbol), a symplectomorphism which simplifies the function may be realized as a conjugation which simplifies the operator. Problems for general operators may thereby be reduced to certain model cases. See [D] for a survey through 1974. A more recent example of this is contained in the work of R. Melrose. In [MEL1], he finds a normal form under symplectomorphisms for pairs of hypersurfaces which are tangent to one another at a point in a certain "nondegenerate" way. This theorem in symplectic geometry can then be applied to the problem of diffraction by a convex obstacle, in which the obstacle is represented by the first hypersurface and a family of light rays by the second. Among the results of this work is a theorem to the effect that, when a ray of light meets an obstacle in a "glancing" way (i.e. tangent to the obstacle at a convex point), the ray continues as if the obstacle were not there-here, a ray represents a path along with the solution is not $C^{\infty}$. (See also [T].) This is in contrast with the situation for the propagation of analyticity where a ray, after grazing an obstacle, produces supplementary rays which "creep" around the boundary of the obstacle and then fly off into space once again at any moment they like. Melrose's approach also leads to very refined results in scattering theory (see [MEL2].)

\section{REFERENCES}

[A-M] R. Abraham and J. Marsden, Foundations of mechanics, 2nd ed., Benjamin/Cummings, Reading, Mass., 1978.

[AR] V. I. Arnold, Mathematical methods of classical mechanics, Graduate Texts in Math., vol. 60, Springer-Verlag, Berlin and New York, 1978.

[AU-K] L. Auslander and B. Kostant, Polarization and unitary representations of solvable Lie groups, Invent. Math. 14 (1971), 255-354. 
[BI] G. D. Birkhoff, Fifty years of American mathematics, Semicentennial Addresses of Amer. Math. Soc.,1938, p. 307.

[BO] C. E. Bond, Biology of fishes, Sanders, Philadelphia, Pa., 1979.

[BU] L. Boutet de Monvel, private communication.

[BU-G] L. Boutet de Monvel and V. Guillemin, The spectral theory of Toeplitz operators, preprint.

[C] S. S. Chern, From triangles to manifolds, Amer. Math. Monthly 86 (1979), 339-349.

[D] J. J. Duistermaat, Applications of Fourier integral operators, Proc. Internat. Congr. Math. (Vancouver, 1974), Canadian Mathematical Congress, 1975, pp. 263-268.

[D-H] J. J. Duistermaat and L. Hörmander, Fourier integral operators. II, Acta Math. 128 (1972), 183-269.

[F-Z] L. Faddeev and V. Zaharov, Korteweg-de Vries equation: a completely integrable Hamiltonian system, Funkcional. Anal. i Priložen 5 (1971), 18-27.

[G] V. W. Guillemin, Clean intersection theory and Fourier integral operators, Lecture Notes in Math., vol. 459, Springer-Verlag, Berlin and New York, 1975, pp. 23-35.

[G-S1] V. W. Guillemin and S. Sternberg, Geometric asymptotics, Amer. Math. Soc., Providence, R. I., 1976.

[G-S2] Some problems in integral geometry and some related problems in micro-local analysis, Amer. J. Math. 101 (1979), 915-955.

[H-K] P. de la Harpe and M. Karoubi, Perturbations compactes des representations d'un groupe dans un espace de Hilbert, Bull. Soc. Math. France Mém. 46 (1976), 41-65.

[HR] L. Hörmander, Fourier integral operators. I, Acta Math. 127 (1971), 79-183.

[K-K-S] D. Kazhdan, B. Kostant, and S. Sternberg, Hamiltonian group actions and dynamical systems of Calogero Type, Comm. Pure Appl. Math. 31 (1978), 481-508.

[KI1] A. A. Kirillov, Unitary representations of nilpotent Lie groups, Uspehi Mat. Nauk. 17 (1962), 57-110. (English translation in Russian Math. Surveys, 53, 104).

[KI2] _ Elements of the theory of representations, Springer-Verlag, Berlin and New York, 1976.

[KO1] B. Kostant, Quantization and unitary representations: Part I, Prequantization, Lecture Notes in Math., vol. 170, Springer-Verlag, Berlin and New York, 1970, pp. 87-208.

[KO2] Math. 34 (1979), 195-338.

[LA1] J. L. Lagrange, Mémoire sur la théorie des variations des éléments des planètes, Mémoires de la classe des sciences mathématiques et physiques de l'institut de France, 1808, pp. 1-72.

[LA2] __ Second mémoire sur la théorie de la variation des constantes arbitraires dans les problèmes de mécanique, Mémoires de la classe des sciences mathématiques et physiques de l'institut de France 1809, pp. 343-352.

[LI] R. G. Littlejohn, $A$ guiding center Hamiltonian: $A$ new approach, J. Math. Phys. 20 (1979), 2445-2458.

[M] G.-M. Marle, Symplectic manifolds, dynamical groups, and Hamiltonian mechanics, in Differential Geometry and Relativity (Cahen and Flato (eds.)), D. Reidel, Dordrecht, 1976, pp. 249-269.

[MA-W] J. Marsden and A. Weinstein, Reduction of symplectic manifolds with symmetry, Reports on Math. Phys. 5 (1974), 121-130.

[MAS] V. P. Maslov, Théorie des perturbations et méthodes asymptotiques, Dunod, Gauthier-Villars, Paris, 1972. (Translation of 1965 Russian edition.)

[MEL1] R. B. Melrose, Equivalence of glancing hypersurfaces, Invent. Math. 37 (1976), 165191.

[MEL2] __ Forward scattering by a convex obstacle, Comm. Pure Appl. Math. 33 (1980), 461-499.

[MEY] K. Meyer, Symmetries and integrals in mechanics, in Dynamical Systems (M. Peixoto (ed)), Academic Press, New York, 1973, pp. 259-273.

[MI-F] A. S. Miščnko and A. T. Fomenko, Euler equations on finite-dimensional Lie groups, Math. USSR-Izv. 12 (1978), 371-389.

[MO] J. Moser, A fixed point theorem in symplectic geometry, Acta Math. 141 (1978), 17-34.

[R-W] L. Rothschild and J. Wolf, Representations of semi-simple groups associated to nilpotent 
orbits, Ann. Sci. École Norm. Sup. (4) 7 (1974), 155-174.

[S-K-K] M. Sato, T. Kawai and M. Kashiwara, Microfunctions and partial differential equations, Lecture Notes in Math., vol. 287, Springer-Verlag, Berlin and New York, 1973, pp. 265-529.

[SE] I. E. Segal, Quantization of nonlinear systems, J. Math. Phys. 1 (1960), 468-488.

[SI] B. Simon, The classical limit of quantum partition functions, Comm. Math. Phys. 71 (1980), 247-276.

[SN] J. Sniatycki, Geometric quantization and quantum mechanics, Springer-Verlag, New York, 1980.

[SN-T] J. Sniatycki and W. M. Tulczyjew, Generating forms of lagrangian submanifolds, Indiana Univ. Math. J. 22 (1972), 267-275.

[SO] J.-M. Souriau, Structure des systemes dynamiques, Dunod, Paris, 1970.

[ST] S. Sternberg, Celestial mechanics. II, W. A. Benjamin, New York, 1969.

[T] M. E. Taylor, Grazing rays and reflection of singularities of solutions to wave equations, Comm. Pure Appl. Math. 24 (1976), 1-38.

[WA] N. R. Wallach, Symplectic geometry and Fourier analysis, Math. Sci. Press, Brookline, Mass., 1977.

[WE1] A. Weinstein, On Maslov's quantization condition, Lecture Notes in Math., vol. 459, Springer-Verlag, Berlin and New York, 1975, pp. 341-372.

[WE2] __ Fourier integral operators, quantization, and the spectra of riemannian manifolds, Colloques Internationaux du CNRS 237 (1976), 289-298.

[WE3] _ Lectures on symplectic manifolds, CBMS Regional Conf. Series, no. 29, Amer. Math. Soc., Providence, R. I., 1977.

[WE4] _ The symplectic "category," Proc. Conf. Differential Geometric Methods in Mathematical Physics (Clausthal-Zellerfeld, 1980) (in preparation).

[WL] H. Weyl, The classical groups, Princeton Univ. Press, Princeton, N. J., 1946.

Department of Mathematics, California institute of Technology, Pasadena, CaliFORNIA 91106

Department of Mathematics, University of California, Berkeley, California 94720 
